\title{
ON THE REIDEMEISTER TORSION OF RATIONAL HOMOLOGY SPHERES
}

\author{
LIVIU I. NICOLAESCU
}

(Received 26 June 2000 and in revised form 21 September 2000)

\begin{abstract}
We prove that the $\bmod \mathbb{Z}$ reduction of the Reidemeister torsion of a rational homology 3-sphere is naturally a $\mathbb{Q} / \mathbb{Z}$-valued quadratic function uniquely determined by a $\mathbb{Q} / \mathbb{Z}$-constant and the linking form.

2000 Mathematics Subject Classification. Primary 57M27, 57Q10.
\end{abstract}

1. Introduction. Recently, V. Turaev has proved in [3, Theorem 4.3.1] a certain identity involving the Reidemeister torsion of a rational homology sphere $M$. In this paper, we suitably interpret this identity as a second-order finite difference equation satisfied by the torsion. Roughly speaking this identity states that the finite difference Hessian of the torsion coincides with the linking form of $M$. This allows us to prove a general structure result for the $\bmod \mathbb{Z}$ reduction of the torsion. More precisely, in Proposition 3.3 we prove that the $\bmod \mathbb{Z}$ reduction of the torsion is completely determined by three data.

- a certain canonical $\operatorname{spin}^{c}$-structure $\sigma_{0}$,

- the linking form lk of $M$,

- a constant $c \in \mathbb{Q} / \mathbb{Z}$.

By fixing the $\operatorname{spin}^{c}$-structure $\sigma_{0}$, we have a natural choice of Euler structure and thus, we can identify the Reidemeister torsion with a $\mathbb{Q}$-valued function on $H:=H_{1}(M, \mathbb{Z})$. Its $\bmod \mathbb{Z}$ reduction is a function $\tau: H \rightarrow \mathbb{Q} / \mathbb{Z}$ of the form

$$
\tau(h)=c-\widehat{\mathbf{l k}}(h),
$$

where $\widehat{\mathbf{l k}}$ denotes a quadratic form on $H$ such that

$$
\widehat{\mathbf{l k}}\left(h_{1}+h_{2}\right)-\widehat{\mathbf{l k}}\left(h_{1}\right)-\widehat{\mathbf{l k}}\left(h_{2}\right)=\mathbf{l k}\left(h_{1}, h_{2}\right) .
$$

As a consequence, the constant $c$ is a $\mathbb{Q} / \mathbb{Z}$-valued invariant of the rational homology sphere. Experimentations with lens spaces suggest this invariant is as powerful as the torsion itself.

2. The Reidemeister torsion. We review briefly a few basic facts about the Reidemeister torsion of a rational homology 3-sphere. For more details and examples we refer to [1, 3]. 
Suppose that $M$ is a rational homology sphere. We set $H:=H_{1}(M, \mathbb{Z})$ and use the multiplicative notation to denote the group operation on $H$. To remove the sign ambiguities in the definition of torsion, we equip $H_{*}(M, \mathbb{R})$ with the canonical orientation described in [3].

Denote by $\operatorname{Spin}^{c}(M)$ the set of isomorphism classes of $\operatorname{spin}^{c}$-structure on $M$. It is an $H$-torsor, that is, the group $H$ acts freely and transitively on $\operatorname{Spin}^{c}(M)$,

$$
H \times \operatorname{Spin}^{c}(M) \ni(h, \sigma) \longmapsto h \cdot \sigma \in \operatorname{Spin}^{c}(M) .
$$

We denote by $\mathscr{F}_{M}$ the space of functions

$$
\phi: H \longrightarrow \mathbb{Q} \text {. }
$$

The group $H$ acts on $\mathscr{F}_{M}$ by

$$
H \times \mathscr{F}_{M} \ni(\mathfrak{g}, \phi) \longmapsto g \cdot \phi,
$$

where

$$
(g \cdot \phi)(h)=\phi(h g)
$$

We denote by $\int_{H}$ the augmentation map

$$
\mathscr{F}_{M} \longrightarrow \mathbb{Q}, \quad \int_{H} \phi:=\sum_{h \in H} \phi(h) .
$$

According to [3], the Reidemeister torsion is an $H$-equivariant map

$$
\tau: \operatorname{Spin}^{c}(M) \longrightarrow \mathscr{F}_{M}, \quad \operatorname{Spin}^{c}(M) \ni \sigma \longmapsto \tau_{\sigma}=\tau_{M, \sigma} \in \mathscr{F}_{M}
$$

such that

$$
\int_{H} \tau_{\sigma}=0
$$

In particular, if $M$ is an integral homology sphere we have $\tau_{M, \sigma}=0$. Denote by $\mathbf{l k}_{M}$ the linking form of $M$,

$$
\mathbf{l k}_{M}: H \times H \longrightarrow \mathbb{Q} / \mathbb{Z} .
$$

V. Turaev has proved in [3] that $\tau_{\sigma}$ satisfies the identity

$$
\boldsymbol{\tau}_{\sigma}\left(g_{1} g_{2}\right)-\boldsymbol{\tau}_{\sigma}\left(g_{1}\right)-\boldsymbol{\tau}_{\sigma}\left(g_{2}\right)+\boldsymbol{\tau}_{\sigma}(1)=-\mathbf{l k}_{M}\left(g_{1}, g_{2}\right) \bmod \mathbb{Z}
$$

for all $g_{1}, g_{2} \in H, \sigma \in \operatorname{Spin}^{c}(M)$. In the above identity, we replace $\sigma$ by $h \cdot \sigma$ for an arbitrary $h \in H$ and using the $H$-equivariance of $\sigma \mapsto \tau_{\sigma}$, we deduce

$$
\boldsymbol{\tau}_{\sigma}\left(g_{1} g_{2} h\right)-\tau_{\sigma}\left(g_{1} h\right)-\tau_{\sigma}\left(g_{2} h\right)+\tau_{\sigma}(h)=-\mathbf{l k}_{M}\left(g_{1}, g_{2}\right) \bmod \mathbb{Z}
$$

for all $g_{1}, g_{2}, h \in H, \sigma \in \operatorname{Spin}^{c}(M)$. 
3. A second-order differential equation. The identity (2.10) admits a more suggestive interpretation. To describe it, we need a few more notation.

Denote by $\mathscr{Y}_{M}$ the space of functions $H \rightarrow \mathbb{Q} / \mathbb{Z}$. Each $g \in H$ defines a first-order differential operator

$$
\Delta_{g}: \mathscr{Y}_{M} \longrightarrow \mathscr{S}_{M}, \quad\left(\Delta_{g} u\right)(h):=u(g h)-u(h), \quad \forall u \in \mathscr{Y}_{M}, h \in H .
$$

If $\Xi=\Xi_{\sigma}$ denotes the $\bmod \mathbb{Z}$ reduction of $\boldsymbol{\tau}_{\sigma}$, then we can rewrite (2.10) as

$$
\left(\Delta_{g_{1}} \Delta_{g_{2}} \Xi\right)(h)=-\mathbf{l k}_{M}\left(g_{1}, g_{2}\right) .
$$

Note that the second-order differential operator $\Delta_{g_{1}} \Delta_{g_{2}}$ can be regarded as a sort of Hessian.

We prove uniqueness and existence results for this equation. We begin with the (almost) uniqueness part.

LEMMA 3.1. The second-order linear differential equation (3.2) determines $\Xi$ up to an "affine" function, that is, the sum between a character of $H$ and $a \mathbb{Q} / \mathbb{Z}$-constant.

Proof. Suppose that $\Xi_{1}, \Xi_{2}$ are two solutions of the above equation. Set $\Psi:=$ $\Xi_{1}-\Xi_{2}, \Psi$ satisfies the equation

$$
\Delta_{g_{1}} \Delta_{g_{2}} \Psi=0
$$

Now, observe that any function $F \in \mathscr{Y}_{M}$ satisfying the second-order equation

$$
\Delta_{u} \Delta_{v} F=0, \quad \forall u, v \in H
$$

is affine, that is, it has the form

$$
F=c+\lambda,
$$

where $c \in \mathbb{Q} / \mathbb{Z}$ is a constant and $\lambda: H \rightarrow \mathbb{Q} / \mathbb{Z}$ is a character. Indeed, the condition

$$
\Delta_{u}\left(\Delta_{v} F\right)=0, \quad \forall u
$$

implies $\Delta_{v} F$ is a constant depending on $v, c(v)$. Thus

$$
F(v h)-F(h)=c(v), \quad \forall h .
$$

The function $\lambda=F-F(1)$ satisfies the same differential equation

$$
\lambda(v h)-\lambda(h)=c(v)
$$

and the additional condition $\lambda(1)=0$. If we set $h=1$ in the above equation, we deduce

$$
\lambda(v)=c(v)
$$

Hence,

$$
\lambda(v h)=\lambda(h)+\lambda(v), \quad \forall v, h
$$

so that $\lambda$ is a character of $H$ and $F=F(1)+\lambda$. Thus, the differential equation (3.2) determines $\Xi$ up to a constant and a character. 
LEMмA 3.2. Suppose that $b: H \times H \rightarrow \mathbb{Q} / \mathbb{Z}$ is a nonsingular, symmetric, bilinear form on $H$. Then there exists a quadratic form $q: H \rightarrow \mathbb{Q} / \mathbb{Z}$ such that

$$
\mathscr{H} q=b,
$$

where

$$
(\mathscr{H} q)(u, v):=q(u v)-q(u)-q(v) .
$$

Proof. Let us briefly recall the terminology in this lemma. $b$ is nonsingular if the induced map $H \rightarrow H^{\sharp}:=\operatorname{Hom}(H, \mathbb{Q} / \mathbb{Z})$ is an isomorphism. A quadratic form is a function $q: H \rightarrow \mathbb{Q} / \mathbb{Z}$ such that

$$
q(1)=0, \quad q\left(u^{k}\right)=k^{2} q(u), \quad \forall u \in H, k \in \mathbb{Z}
$$

and $\mathscr{H} q$ is a bilinear form.

Suppose that $b$ is a nonsingular, symmetric, bilinear form $H \times H \rightarrow \mathbb{Q} / \mathbb{Z}$. Then, according to [4, Section 7 ], $b$ admits a resolution. This is a nondegenerate, symmetric, bilinear form

$$
B: \Lambda \times \Lambda \longrightarrow \mathbb{Z}
$$

on a free abelian group $\Lambda$ such that the induced monomorphism $J_{B}: \Lambda \rightarrow \Lambda^{*}:=$ $\operatorname{Hom}(\Lambda, \mathbb{Z})$ is a resolution of $H$,

$$
0 \longleftrightarrow \Lambda \stackrel{J_{B}}{\longrightarrow} \Lambda^{*} \stackrel{\pi}{\longrightarrow} H \longrightarrow 0
$$

and $b$ coincides with the induced bilinear form on $\Lambda^{*} /\left(J_{B} \Lambda\right)(n:=\# H)$,

$$
b(\pi(u), \pi(v))=\frac{1}{n^{2}} B\left(J_{B}^{-1}(n u), J_{B}^{-1}(n v)\right) \bmod \mathbb{Z}, \quad \forall u, v \in \Lambda^{*} .
$$

Now, set

$$
q(\pi(u))=\frac{1}{2 n^{2}} B\left(J_{B}^{-1}(n u), J_{B}^{-1}(n u)\right) \bmod \mathbb{Z} .
$$

This quantity is well defined, that is,

$$
\frac{1}{2 n^{2}} B\left(J_{B}^{-1}(n u), J_{B}^{-1}(n u)\right)=\frac{1}{2 n^{2}} B\left(J_{B}^{-1}(n v), J_{B}^{-1}(n v)\right) \bmod \mathbb{Z}
$$

if $v=u+J_{B} \lambda, \lambda \in \Lambda$. Clearly, $\mathscr{H} q=b$.

Denote by $Q$ the space of solutions of the equation (3.11), that is, the space of quadratic forms $q$ on $H$ satisfying $\mathscr{H} q=-\mathbf{l k}_{M}$. $Q$ consists of more than one element. It is a $G$-torsor, where $G=\operatorname{Hom}\left(H, \mathbb{Z}_{2}\right)$ and the $G$ action is given by

$$
(Q \times G) \ni(q, \mu) \longmapsto q+\mu .
$$

Using the linking form on $M$ we can identify $G$ with the 2-torsion subgroup of $H$. Denote by $\Xi_{\sigma}$ the reduction $\bmod \mathbb{Z}$ of $\boldsymbol{\tau}_{\sigma}$.

Fix a $\operatorname{spin}^{c}$ structure $\sigma_{0}$ on $M$. We deduce that for every $q \in Q$ there exists a constant $k=k(q)$ and a character $\lambda=\lambda_{q}$ of $H$

$$
\Xi_{\sigma_{0}}(h)=k(q)+\lambda_{q}(h)+q(h), \quad \mathscr{H} q=-\mathbf{l k}_{M} .
$$


In particular,

$$
\begin{aligned}
\Xi_{g \cdot \sigma_{0}}(h): & =\Xi_{\sigma}(g h)=k(q)+\lambda_{q}(g h)+q(g h) \\
& =\underbrace{\left(k(q)+\lambda_{q}(g)+q(g)\right)}_{c(g, q)}+\underbrace{\left(\lambda_{q}(h)+(\mathscr{H} q)(g, h)\right)}_{\lambda_{q, g}(h)}+q(h)
\end{aligned}
$$

where $\lambda_{q, g}(\bullet)=\lambda_{q}(\bullet)-\mathbf{l k}_{M}(g, \bullet)$. Since the linking form is nondegenerate we can find a unique $g=g(q)$ such that $\lambda_{q, g}=0$. We set $\vec{\sigma}(q)=g(q) \cdot \sigma_{0}$ and $c(q)=c(g(q), q)$. The above computation also shows that for every $\mu \in G$ we have

$$
c(q+\mu)-c(q)=q(\mu), \quad \vec{\sigma}(q+\mu)=\mu \cdot \vec{\sigma}(q) .
$$

We have thus proved the following result.

Proposition 3.3. Suppose $M$ is a rational homology sphere. Then there exist functions

$$
c: Q \rightarrow \mathbb{Q} / \mathbb{Z}, \quad \vec{\sigma}: Q \rightarrow \operatorname{Spin}^{c}(M)
$$

so that

$$
\tau_{\vec{\sigma}(q)}(h):=q(h)+c(q) \bmod \mathbb{Z}, \quad \forall h \in H .
$$

Moreover,

$$
c(q+\mu)-c(q)=q(\mu), \quad \vec{\sigma}(q+\mu)=\mu \cdot \vec{\sigma}(q), \quad \forall \mu \in G .
$$

REMARK 3.4. (a) Note that $q(\mu) \in(1 / 4) \mathbb{Z}, \forall q \in Q, \mu \in \mathbb{Z}$ so that $4 c(q)$ is independent of $q$. It is a topological invariant of $M$ !

(b) One can show that the image of the one-to-one map $\vec{\sigma}$ is $\operatorname{Spin}(M)$, the set $\operatorname{spin}^{c}$ structures induced by the spin structures on $M$. We can thus regard $c$ as a map $c$ : $\operatorname{Spin}(M) \rightarrow \mathbb{Q} / \mathbb{Z}$.

4. Examples. We want to show on some simple examples that the invariant $c$ is nontrivial. First, we need some notation.

We denote by $\mathbb{Z}_{n}$ the cyclic group with $n$ elements. The functions $f: \mathbb{Z}_{n} \rightarrow \mathbb{Q}$ can be conveniently described as polynomials $f \in \mathbb{Q}[x]$, where $x^{n}=1$. Given two such polynomials $f, g$, we define the equivalence relation $\sim$ by

$$
f \sim g \Longleftrightarrow \exists m \in \mathbb{Z}: f= \pm x^{m} g .
$$

We will not keep track of Euler structures and/or homology orientations and that is why in the sequel only the -equivalence class of the torsion will be well defined. In particular, the map $c$ constructed in the previous section will be defined only up to a sign.

(a) Suppose that $M=L(8,3)$. Then its torsion is (see [2])

$$
T_{8,3} \sim-\frac{9}{32} x^{7}-\frac{3}{32} x^{6}-\frac{9}{32} x^{5}+\frac{5}{32} x^{4}+\frac{7}{32} x^{3}-\frac{3}{32} x^{2}+\frac{7}{32} x+\frac{5}{32},
$$

where $x^{8}=1$ is a generator of $\mathbb{Z}_{8}$. Then

$$
q\left(x^{n}\right)=\frac{-3 n^{2}}{16} .
$$


The set of possible values $\left(-3 m^{2} / 16\right) \bmod \mathbb{Z}$ is

$$
A:=\left\{0, \frac{-3}{16}, \frac{4}{16}, \frac{5}{16}\right\} .
$$

The set of possible values of $\Xi(h)$ is

$$
B:=\left\{-\frac{9}{32},-\frac{3}{32}, \frac{5}{32}, \frac{7}{32}\right\} .
$$

We need to find a constant $c \in \mathbb{Q} / \mathbb{Z}$ such that

$$
B \pm c=A
$$

Equivalently, we need to figure out orderings $\left\{a_{1}, a_{2}, a_{3}, a_{4}\right\}$ and $\left\{b_{1}, b_{2}, b_{3}, b_{4}\right\}$ of $A$ and $B$ such that $b_{i}-a_{i} \bmod \mathbb{Z}$ is a constant independent of $i$. A little trial and error shows that

$$
\vec{A}=\left(0,-\frac{3}{16}, \frac{4}{16}, \frac{5}{16}\right), \quad \vec{B}=\left(-\frac{3}{32},-\frac{9}{32}, \frac{5}{32}, \frac{7}{32}\right)
$$

and the constant $c=-3 / 32$. This is the coefficient of $x^{2}$. We deduce that (modulo $\mathbb{Z}$ )

$$
F:=T_{8,3}(x)+\frac{3}{32} \sim-\frac{3}{16} x^{7}-0 \cdot x^{6}-\frac{3}{16} x^{5}+\frac{1}{4} x^{4}+\frac{1}{4} x^{3}-0 \cdot x^{2}+\frac{1}{4} x+\frac{1}{4} .
$$

The translation of $F$ by $x^{-2}$ is

$$
x^{-2}\left(T_{8,3}+\frac{3}{32}\right)=\frac{1}{4} x^{7}+\frac{1}{4} x^{6}-\frac{3}{16} x^{5}-\frac{3}{16} x^{3}+\frac{1}{4} x^{2}+\frac{1}{4} x
$$

(b) Suppose that $M=L(7,2)$. Then, its torsion is (see [2])

$$
T_{7,2} \sim-\frac{2}{7} x^{6}+\frac{1}{7} x^{5}+\frac{2}{7} x^{3}+\frac{1}{7} x-\frac{2}{7}
$$

where $x^{7}=1$ is a generator of $\mathbb{Z}_{7}$. We see that in this form $T_{7,2}$ is symmetric, that is, the coefficient of $x^{k}$ is equal to the coefficient of $x^{6-k}$. The constant $c$ in this case must be the coefficient of the middle monomial $x^{3}$, which is $2 / 7$.

(c) Suppose that $M=L(7,1)$. Then

$$
T_{7,1} \sim \frac{2}{7} x^{6}+\frac{1}{7} x^{5}-\frac{1}{7} x^{4}-\frac{4}{7} x^{3}-\frac{1}{7} x^{2}+\frac{1}{7} x+\frac{2}{7} .
$$

This is again a symmetric polynomial and the coefficient of the middle monomial is $-4 / 7$. We see that this invariant distinguishes the lens spaces $L(7,1)$ and $L(7,2)$. It is known that these two spaces are homotopic but nonhomeomorphic lens spaces. Thus, the invariant $c$ distinguishes their homeomorphism types, just as the torsion does.

(d) For $M=L(9,2)$, we have

$$
T_{9,2} \sim-\frac{10}{27} x^{8}+\frac{2}{27} x^{7}-\frac{1}{27} x^{6}+\frac{8}{27} x^{5}+\frac{2}{27} x^{4}+\frac{8}{27} x^{3}-\frac{1}{27} x^{2}+\frac{2}{27} x-\frac{10}{27} .
$$


Again, this is a symmetric function, that is, the coefficient of $x^{k}$ is equal to the coefficient of $x^{8-k}, x^{9}=1$. The constant is the coefficient of $x^{4}$, which is $2 / 27$. We deduce that $\bmod \mathbb{Z}$, we have

$$
T_{9,2}-\frac{2}{27}=-\frac{2}{3} x^{8}-\frac{2}{9} x^{7}-\frac{1}{3} x^{6}-\frac{2}{9} x^{7} .
$$

(e) Finally, when $M=L(9,7)$ we have

$$
T_{9,7} \sim-\frac{8}{27} x^{8}-\frac{2}{27} x^{7}+\frac{10}{27} x^{6}+\frac{1}{27} x^{5}-\frac{2}{27} x^{4}+\frac{1}{27} x^{3}+\frac{10}{27} x^{2}-\frac{2}{27} x-\frac{8}{27}
$$

the polynomial is again symmetric so that the constant $c$ is the coefficient of $x^{4}$ which is $-2 / 27$.

ACKNOWLEDGEMENTS. I want to thank Andrew Ranicki for drawing my attention to the results in [4]. I am indebted to Stephan Stolz for many illuminating discussions, and in particular, for pointing out to me the original erroneous assumption that $Q$ consists of a single element. I also want to thank the referees for their comments and suggestions.

\section{REFERENCES}

[1] I. N. Liviu, Reidemeister torsion, preliminary version, http://www.nd.edu/ ${ }^{\sim}$ Inicolae/, December 1999.

[2] _ Seiberg-Witten theoretic invariants of lens spaces, http://www.arxiv.org/abs/ math.DG/9901071, January 1999, submitted.

[3] V. Turaev, Torsion invariants of $\operatorname{Spin}^{c}$-structures on 3-manifolds, Math. Res. Lett. 4 (1997), no. 5, 679-695. MR 98k:57038. Zbl 891.57019.

[4] C. T. C. Wall, Quadratic forms on finite groups, and related topics, Topology 2 (1963), 281298. MR 28\#133. Zbl 215.39903.

LIVIU I. NicolAESCU: UNIVERSITY OF NOTRE DAME, NotRe DAME, IN 46556, USA

E-mail address: 1nicolae@necke. he1ios.nd.edu

URL: http://www.nd.edu/ ${ }^{\sim}$ Inicolae/ 


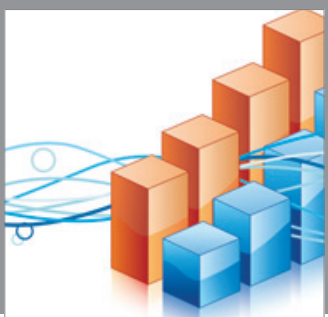

Advances in

Operations Research

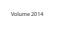

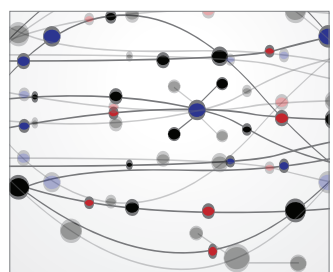

\section{The Scientific} World Journal
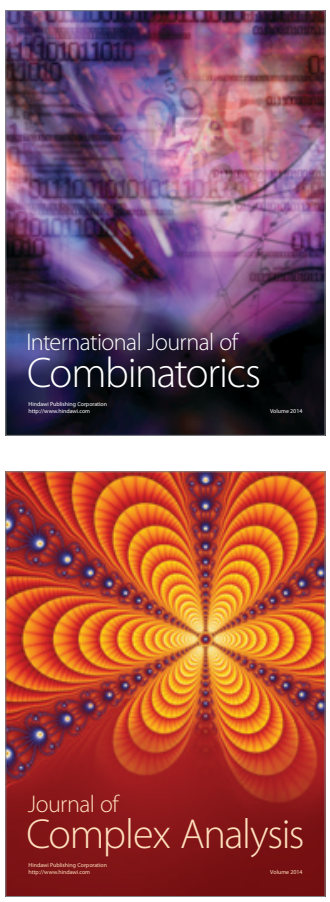

International Journal of

Mathematics and

Mathematical

Sciences
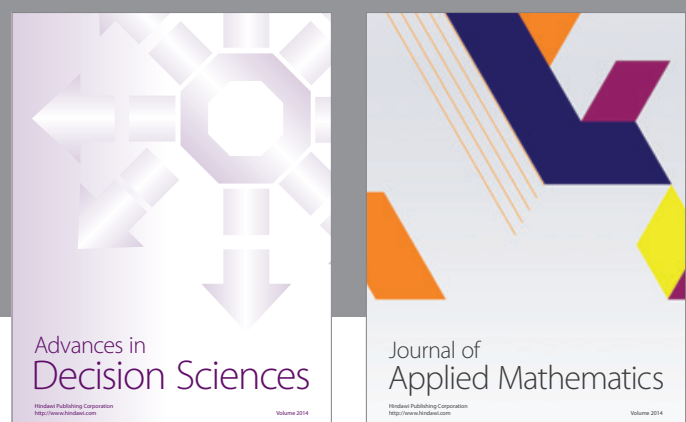

Journal of

Applied Mathematics
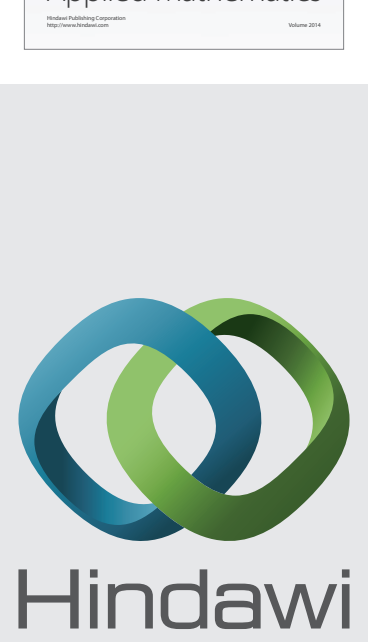

Submit your manuscripts at http://www.hindawi.com
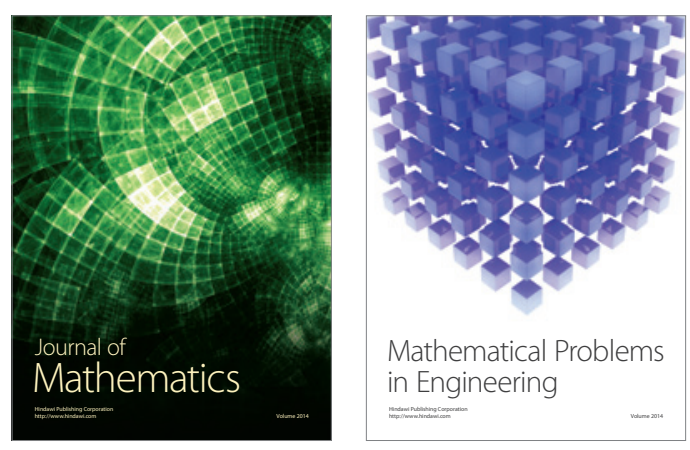

Mathematical Problems in Engineering
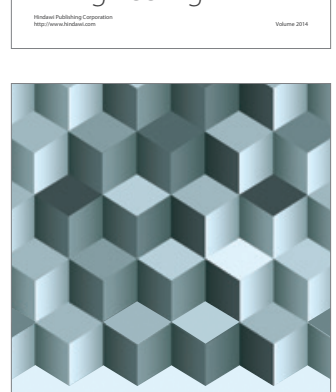

Journal of

Function Spaces
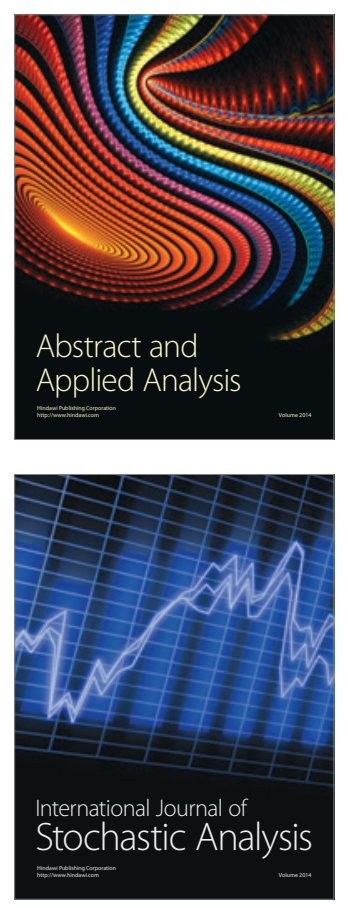

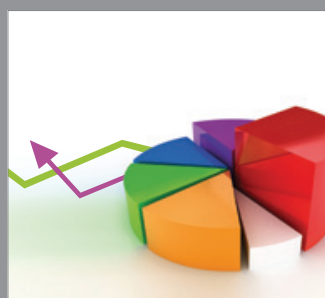

ournal of

Probability and Statistics

Promensencen
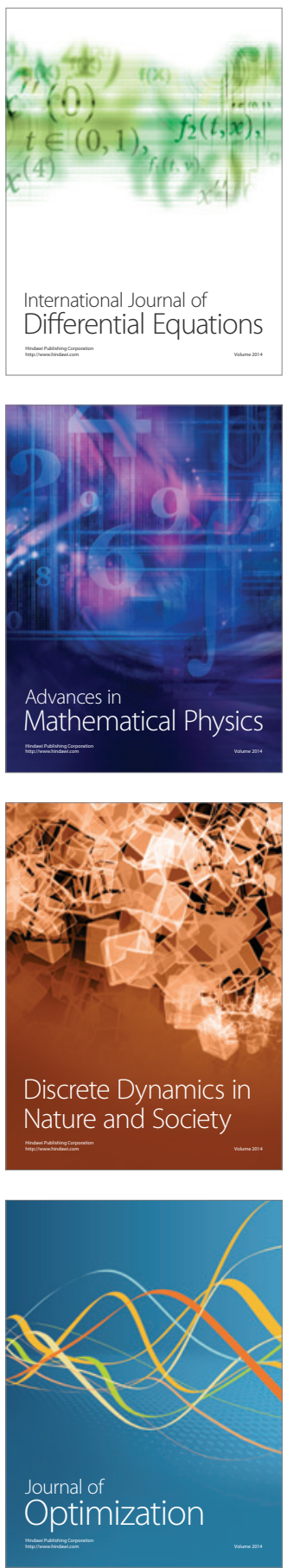